\title{
Systemic tryptophan and kynurenine catabolite levels relate to severity of rhinovirus-induced asthma exacerbation: a prospective study with a parallel-group design
}

\author{
Koenraad F van der Sluijs, ${ }^{1,2,3}$ Marianne A van de Pol, ${ }^{1,2}$ Wim Kulik, $^{4}$ \\ Annemiek Dijkhuis, ${ }^{2}$ Barbara S Smids, ${ }^{1,2}$ Hetty W van Eijk, ${ }^{5}$ Jos A Karlas, ${ }^{5}$ \\ Richard Molenkamp, ${ }^{5}$ Katja C Wolthers, ${ }^{5}$ Sebastian L Johnston, ${ }^{6}$ \\ Jaring $S$ van der Zee, ${ }^{1,7}$ Peter J Sterk, ${ }^{1}$ René Lutter, ${ }^{1,2}$ the RESOLVE research team
}

- Additional material is published online only. To view please visit the journal online (http://dx.doi.org/10.1136/ thoraxjnl-2013-203728).

For numbered affiliations see end of article.

\section{Correspondence to} Dr K F van der Sluiijs, Department of Laboratory of Experimental Intensive Care and Anesthesiology, P.O. Box 22700, Amsterdam 1100 DD, The Netherlands;

kvandersluijs@amc.uva.nl

Received 15 April 2013 Revised 21 June 2013 Accepted 30 June 2013 Published Online First 23 July 2013

\begin{tabular}{l}
\hline To cite: van der Sluijs KF, \\
van de Pol MA, Kulik W, \\
et al. Thorax \\
2013;68:1122-1130. \\
\hline
\end{tabular}

\section{ABSTRACT \\ Background Patients with allergic asthma have exacerbations which are frequently caused by rhinovirus infection. The antiviral tryptophan-catabolising enzyme indoleamine 2,3-dioxygenase (IDO) is induced by interferon- $\gamma$ and suppressed by Th2 mediators interleukin (IL)-4 and IL-13. We hypothesised that local IDO activity after viral airway infection is lower in patients with allergic asthma than in healthy controls. \\ Objective To determine whether IDO activity differs between patients with allergic asthma and healthy individuals before and after rhinovirus infection.}

Methods Healthy individuals and patients with allergic asthma were experimentally infected with low-dose (10 TCID 50 ) rhinovirus 16. Blood, bronchoalveolar lavage fluid and exhaled breath condensate (for mass spectrometry by UPLC-MS/MS) were obtained before and after rhinovirus challenge.

Results IDO activity was not induced by rhinovirus infection in either group, despite increases in cold scores. However, baseline pulmonary IDO activity was lower in patients with allergic asthma than in healthy individuals. In contrast, systemic tryptophan and its catabolites were markedly higher in patients with allergic asthma. Moreover, systemic quinolinic acid and tryptophan were associated with eosinophil cationic protein ( $r=0.43$ and $r=0.78$, respectively) and eosinophils ( $r=0.38$ and $r=0.58$, respectively) in bronchoalveolar lavage fluid and peak asthma symptom scores after rhinovirus challenge $(r=0.53$ and $r=0.64$, respectively).

Conclusions Rhinovirus infection by itself induces no IDO activity, but the reduced pulmonary IDO activity in patients with allergic asthma at baseline may underlie a reduced control of viral infections. Notably, the enhanced systemic catabolism of tryptophan in patients with allergic asthma was strongly related to the outcome of rhinovirus challenge in asthma and may serve as a prognostic factor.

\section{INTRODUCTION}

Exacerbations represent a major clinical manifestation of asthma and are characterised by episodes of acute worsening of symptoms such as shortness of breath, cough, wheezing and chest tightness in

\section{Key messages}

What is the key question?

- Is indoleamine 2,3-dioxygenase-mediated tryptophan catabolism different between patients with allergic asthma and healthy individuals and does tryptophan catabolism change upon experimental rhinovirus infection?

What is the bottom line?

- Tryptophan catabolism is different between healthy individuals and patients with allergic asthma, and is associated with symptom scores and eosinophilic inflammation after rhinovirus exposure.

\section{Why read on?}

- Our study shows that local tryptophan catabolism markedly differs from systemic tryptophan catabolism.

conjunction with airways obstruction. ${ }^{1}$ Rhinoviruses are the most prominent pathogens to cause exacerbations in patients with asthma. ${ }^{2-4}$ The underlying mechanisms leading to these lower respiratory tract symptoms are thought to involve both innate and adaptive antiviral immune responses. ${ }^{5}$ In particular, Th1 (interferon (IFN) $\gamma$ ) and Th2 (interleukin (IL)-4, IL-5 and IL-13) cytokines produced by CD4 cells were shown to be associated with, respectively, peak expiratory flow and chest symptoms after experimental rhinovirus infection in patients with asthma. $^{6}$

The expression of the tryptophan-catabolising enzyme indoleamine 2,3-dioxygenase (IDO) is induced by IFN $\gamma$ and inhibited by IL- 4 and IL-13. ${ }^{78}$ Considering the predominant Th2 cytokine profile in the lungs of individuals with asthma, it can be postulated that patients with allergic asthma fail to induce adequate IDO activity upon viral airway infection. Impaired IDO activity may play a critical role during virus-induced asthma exacerbations, since IDO has been shown to posses both antiviral ${ }^{9-11}$ and 
immunosuppressive properties. $^{12}{ }^{13}$ Several studies underline the immunosuppressive role of IDO in models of allergic airway inflammation. A study by Hayashi et $a l^{14}$ indicated that CpG-induced IDO expression in the lungs reduces airway hyperreactivity in mice with experimental asthma. In line with this, Taher $e t a l^{15}$ showed that allergen immunotherapy induces tolerance in mice through an IDO-dependent mechanism. In humans, von Bubnoff et $a l^{16}$ found that asymptomatic allergy was associated with enhanced systemic IDO activity. Whether IDO activity is impaired in the lungs of patients with asthma is unknown.

In the present study we hypothesised that local IDO activity is impaired in patients with allergic asthma after viral airway infection. To that end, we sought to determine: (1) whether IDO-mediated tryptophan catabolism is different between patients with allergic asthma and healthy individuals; and (2) whether IDO-mediated tryptophan catabolism changes upon experimental rhinovirus infection in both healthy individuals and patients with allergic asthma. For this purpose, tryptophan, its product kynurenine and further downstream metabolites anthranilic acid and quinolinic acid were measured in serum and exhaled breath condensate (EBC) before and after experimental rhinovirus infection in healthy individuals and patients with mild allergic asthma.

\section{METHODS}

\section{Subjects}

Healthy individuals met the following criteria: baseline forced expiratory volume in $1 \mathrm{~s}\left(\mathrm{FEV}_{1}\right)>80 \%$ predicted, airway responsiveness to methacholine (provocative concentration causing $20 \%$ fall in $\mathrm{FEV}_{1}, \mathrm{PC}_{20}$ ) $>16 \mathrm{mg} / \mathrm{mL}$, skin prick test negative for 12 common aeroallergens. Patients with mild allergic asthma met the following criteria: a history of episodic chest symptoms, baseline $\mathrm{FEV}_{1}>80 \%$ predicted, $\mathrm{PC}_{20}<8 \mathrm{mg} / \mathrm{mL}$, skin prick test positive for at least one of 12 common aeroallergens. All volunteers were aged between 18 and 40 years, were non-smoking or had stopped smoking $>12$ months ago with $\leq 5$ pack years, were negative for serum neutralising antibodies against rhinovirus 16 and did not have concomitant disease or (chronic) inflammatory condition that would interfere with this study according to the judgement of a pulmonary physician. Volunteers with an underlying viral airway infection on day -1 , as determined by PCR on nasal swabs, were withdrawn from the study. Patients with allergic asthma were not allowed to use asthma medication other than short-acting $\beta_{2}$ agonists within 2 weeks prior to the start of the study until day 6 after rhinovirus infection.

\section{Study design}

Healthy individuals and patients with stable allergic asthma were included in a prospective study with a parallel-group design (figure 1). Between March 2009 and June 2011, 14 healthy individuals and 14 patients with allergic asthma were experimentally infected with low-dose $\left(10\right.$ TCID $\left._{50}\right)$ rhinovirus 16 (RV16) as described elsewhere. ${ }^{17}$ In brief, snap-frozen RV16 was rapidly defrosted and taken up in a total volume of $750 \mu \mathrm{L}$ sterile saline and directly sprayed into one of the nostrils by a DeVilbiss atomiser (DeVilbiss 286; DeVilbiss, Somerset, Pennsylvania, USA). All volunteers were requested to report common cold (sneezing, nasal discharge, stuffy nose, sore throat, cough, chest pain, fever, chills and headache) and asthma symptoms (breathlessness, wheezing, cough, chest tightness and increase in asthma symptoms during the night) daily. ${ }^{18}$ Individual symptoms were scored on the basis of severity $(0=$ absent, $1=$ mild, $2=$ moderate, $3=$ severe). In addition, volunteers were also asked to report $\mathrm{FEV}_{1}$ as measured twice daily by a portable spirometer (Piko-1, Ferraris Respiratory Europe, UK) and use of short-acting $\beta_{2}$ agonists. Diaries and portable spirometers were collected at the final visit (between days 42 and 56).

\section{Sample collections}

One day before and 6 days after exposure to RV16, nasal swabs and venous blood were collected from all volunteers. In addition, bronchoalveolar lavage (BAL) fluid and bronchial brushes were collected by a standardised bronchoscopic procedure. ${ }^{19}$

Rhinovirus PCR and neutralisation assay

Nasal swabs, bronchial brushes and $300 \mu \mathrm{L}$ aliquots of BAL fluid and resuspended BAL cells were processed for rhinovirus detection by quantitative PCR as described elsewhere. ${ }^{20}$ For RV16 neutralisation, HEL cells were infected with 100 TCID $_{50}$ RV16 and cultured in the presence of serially diluted serum obtained at the screening visit or between days 42 and day 56 after RV16 exposure. The presence of cytopathic effect in cell cultures was determined 10 days later. The Reed-Muench method was used to estimate neutralisation titre. ${ }^{21}$ Volunteers who were PCR-negative for rhinovirus on day 6 and did not show increased serum neutralisation after the final visit were excluded from the analysis.

\section{Analyses of inflammatory parameters}

IL-4, IL-5, IL-8, IL-13 and IFN $\gamma$ were determined by Luminex according to the manufacturer's protocol (Bio-Rad Laboratories, Veenendaal, The Netherlands). Eosinophil cationic protein (ECP) and myeloperoxidase (MPO) were measured by ELISA as described previously. ${ }^{22}$ Cells in BAL fluid were counted and differentiated using cytospin preparations. Fractional exhaled nitric oxide (FeNO) was measured by a chemiluminescence analyser (Niox Aerocrine AB, Solna, Sweden). ${ }^{23}$
Figure 1 Study design. Screening was performed $14-56$ days before experimental rhinovirus infection. Venous blood, bronchoalveolar lavage fluid and exhaled breath condensate (EBC) were collected at the indicated time points before and after rhinovirus challenge. Blood to determine rhinovirus neutralisation was collected between days 42 and 56 after exposure to rhinovirus type 16 (RV16).

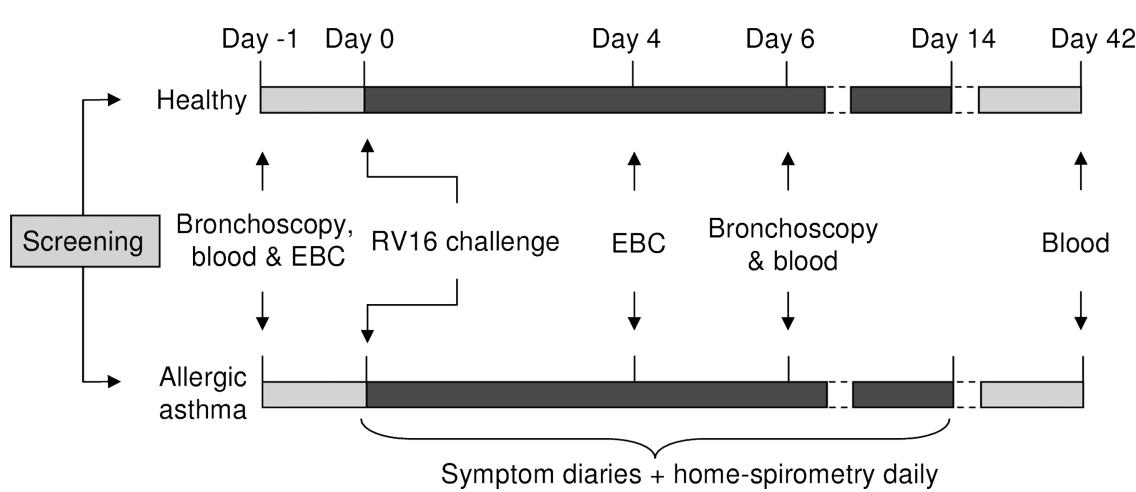


Exhaled breath condensate (EBC)

EBC was collected 1 day before and 4 days after rhinovirus infection using the ECOScreen 1 (Erich Jaeger, Hoechberg, Germany) following the European Respiratory Society Methodological Recommendations Task Force. ${ }^{24}$ The EBC was stored at $-80^{\circ} \mathrm{C}$.

\section{Measurements}

Analyses of serum and EBC were performed by ultraperformance liquid chromatography hyphenated to tandem mass spectrometry (UPLC-MS/MS). In addition to tryptophan and metabolites of the kynurenine pathway, we also included arginine and related metabolites in our analysis since Lara et $a l^{25}$ reported differences in arginine metabolome between healthy individuals and those with asthma. A mix of stable isotopicallylabelled internal standards was added to $0.5 \mathrm{~mL}$ EBC. The sample was dried and reconstituted in $100 \mu \mathrm{L}$ of eluent and $20 \mu \mathrm{L}$ was injected in the UPLC-MS/MS. The UPLC-MS/MS is an Acquity-Quattro Premier XE system (Waters, Milford, Massachusetts, USA) operated in the positive ESI mode using MRMs for the preselected analytes and an overall run time of 12 min.

\section{Statistical analysis}

The sample size was estimated based on cytokine production in previous studies with RV16, ${ }^{26}$ showing variation of $20 \%$ after RV16 infection in patients with allergic asthma. Fourteen individuals per group were needed to detect an effect of $25 \%$ with two-sided significance of $\mathrm{p}=0.05$ and a power of $90 \%$.

Demographic data were compared by the Mann-Whitney $\mathrm{U}$ test or Fisher exact test where appropriate. Variables that were measured on a daily basis were analysed by two-way ANOVA with Bonferroni post hoc test. Differences between time points were analysed by the Wilcoxon matched pairs test and differences between patient groups were analysed by the MannWhitney U test. For tryptophan and related metabolites we used a Bonferroni-adjusted $\mathrm{p}$ value, where tryptophan was considered to be an independent variable and kynurenine, anthranilic acid and quinolinic acid were dependent variables. Serum and EBC were considered as independent compartments. Due to possible disparity between the site of infection and the site of sampling, we performed post hoc analysis for tryptophan metabolism in subjects with PCR-confirmed lower respiratory tract infection. In addition, we performed post hoc analysis for markers of arginine metabolism. All other parameters were predefined as primary or secondary endpoints. Spearman correlation was used to determine associations between amino acid levels, symptom scores and inflammatory markers; $\mathrm{p}<0.05$ was considered significant.

\section{RESULTS}

After obtaining informed consent, 108 volunteers were screened, of whom 73 were not eligible for the study because of pre-existing RV16 antibodies or not fulfilling criteria for asthma (figure 2). Thirty-five subjects (17 healthy individuals and 18 with asthma) were enrolled. Three healthy individuals and four patients with allergic asthma were withdrawn from the study after the first bronchoscopy. The baseline characteristics are shown in table 1.

\section{Virology}

On day 6, nine healthy individuals (64\%) and 11 patients with allergic asthma (79\%) had a PCR-confirmed infection. The virus load did not differ significantly between the groups (figure 3A). Nine healthy individuals (64\%) and 11 patients with allergic asthma (79\%) had RV16-neutralising antibodies in the serum collected at the final visit (table 1). Three healthy individuals and one patient with allergic asthma were excluded (figure 2).

\section{Symptoms}

Both healthy individuals and those with asthma showed a significant increase in common cold score after experimental infection with a low dose of rhinovirus (figure $3 \mathrm{~B}$ ), with the maximum increase on day 6 (ANOVA: $\mathrm{p}<0.05$ for both groups). Although common cold symptoms appeared higher for patients with allergic asthma at baseline, common cold scores did not differ significantly between healthy individuals and patients with allergic asthma throughout the course of infection.

Experimental rhinovirus infection in patients with allergic asthma resulted in an increase in asthma symptoms (figure 3C) which peaked between days 6 and 8 after exposure ( $p<0.05$ vs healthy individuals). Patients with allergic asthma also showed a significant fall in $\mathrm{FEV}_{1}$ (figure 3D).

\section{Inflammation}

ECP levels in BAL fluid were significantly increased after rhinovirus infection in patients with allergic asthma but not in healthy individuals (table 2). FeNO, ECP and eosinophils in BAL fluid were significantly higher in patients with allergic asthma than in healthy individuals at day 1 before and day 6 after rhinovirus exposure (table 2). IL-8, MPO and BAL fluid cells other than eosinophils were not significantly different between the two time points and between the two groups (table 2). IL-4, IL-5, IL-13 and IFN $\gamma$ were undetectable in BAL fluid.

\section{Local and systemic amino acid metabolism}

Patients with allergic asthma showed significantly higher levels of tryptophan, kynurenine, quinolinic acid and anthranilic acid in serum than healthy individuals (figure 4) and were not affected by exposure to RV16. In contrast, patients with allergic asthma showed lower levels of kynurenine (day -1), anthranilic acid (day -1) and quinolinic acid (day 4) in EBC than healthy individuals (figure 4). Tryptophan levels in EBC were not different between healthy individuals and patients with allergic asthma. Similar differences were observed when subjects without PCR-confirmed lower respiratory tract infections were excluded (see online supplementary figure S1).

Post hoc analysis revealed significantly higher serum arginine levels for patients with allergic asthma than for healthy individuals, which was not affected by rhinovirus infection (see online supplementary figure S2). Patients with allergic asthma had lower levels of arginine and markedly higher levels of urea in EBC than healthy individuals, while lower ornithine levels in EBC were lower after rhinovirus infection $(\mathrm{p}<0.05$ vs healthy individuals).

\section{Tryptophan catabolism and rhinovirus-induced exacerbation}

Quinolinic acid was significantly lower in EBC from patients with allergic asthma than healthy individuals after rhinovirus challenge. However, quinolinic acid levels in EBC did not show an association with virus load, asthma symptom scores or eosinophilic inflammation in the lung. Systemic levels of quinolinic acid were associated with peak asthma symptom scores (Spearman $\mathrm{r}=0.5298, \mathrm{p}=0.0078$ ) and ECP in BAL fluid $(\mathrm{r}=0.4321, \mathrm{p}=0.0395)$. The associations of systemic tryptophan with peak asthma symptom scores $(r=0.6421, \mathrm{p}=0.0007)$, percentage BAL fluid eosinophils $(r=0.5840, p=0.0027)$ and ECP 
Figure 2 Flowchart for inclusion of healthy individuals and patients with allergic asthma. RV16, rhinovirus type $16 ; P C_{20}$, airway responsiveness to methacholine bromide (provocative concentration causing a $20 \%$ fall in $\left.F E V_{1}\right) ; F E V_{1}$, forced expiratory volume in $1 \mathrm{~s}$.

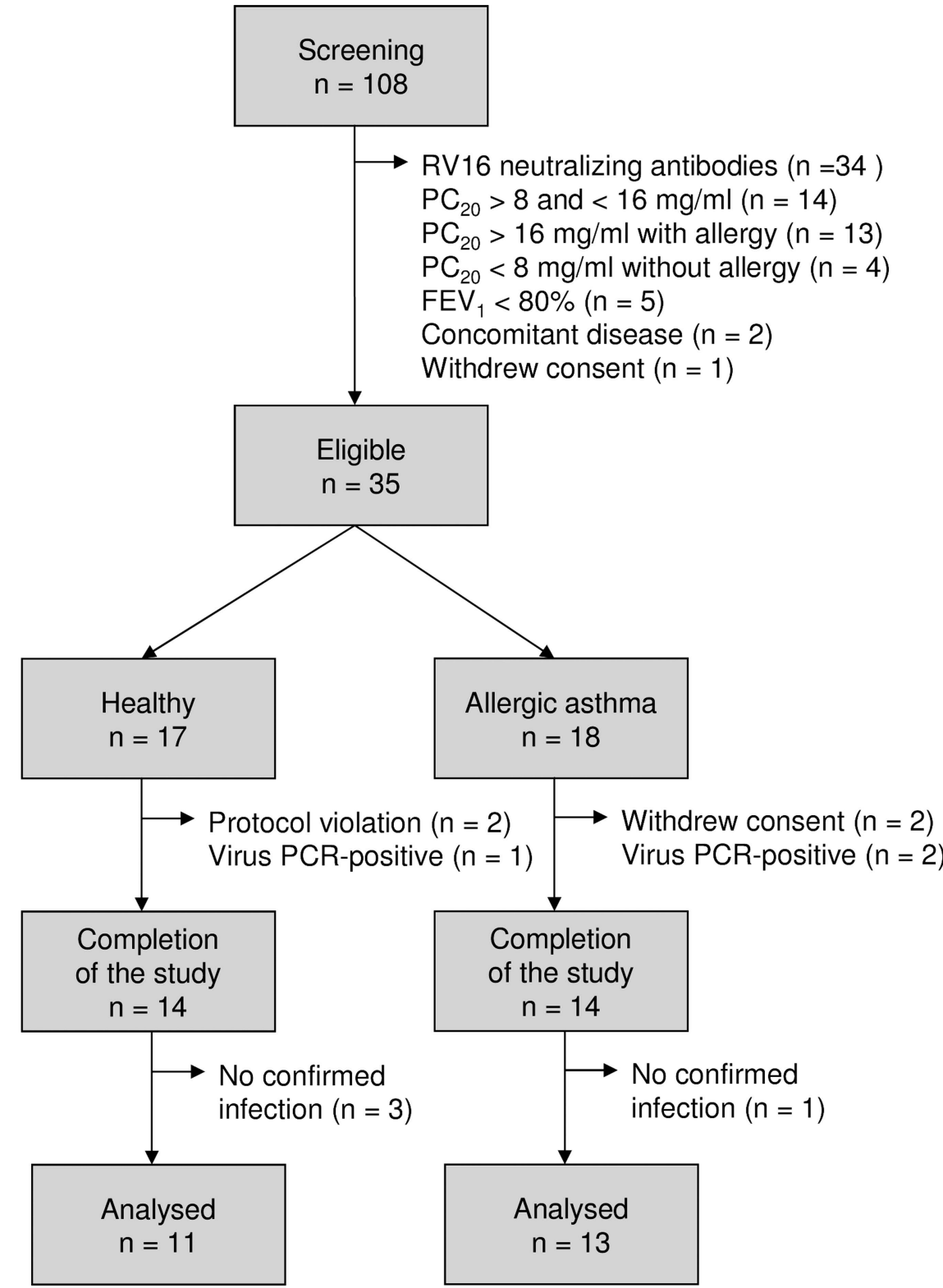

Table 1 Demographic data of study subjects

\begin{tabular}{|c|c|c|}
\hline & Allergic asthma & Healthy \\
\hline $\mathrm{N}$ & 14 & 14 \\
\hline Age, mean (range) & $21.5(19-32)$ & $21(19-31)$ \\
\hline $\operatorname{Sex}(M / F)$ & $7 / 7^{*}$ & $1 / 13$ \\
\hline $\mathrm{FEV}_{1} \%$ pred, mean $\pm \mathrm{SD}$ & $105 \pm 12$ & $110 \pm 12$ \\
\hline $\mathrm{PC}_{20}$, geometric mean $(95 \% \mathrm{Cl})$ & $2.41(1.29 \text { to } 4.48)^{* * *}$ & $>16$ \\
\hline PCR-positive for RV16 on day $6, n$ (\%) & $11(79 \%)$ & $9(64 \%)$ \\
\hline RV16 neutralisation on day $42-56, n(\%)$ & $11(79 \%)$ & $9(64 \%)$ \\
\hline Confirmed viral infectiont, $\mathrm{n}(\%)$ & $13(93 \%)$ & $11(79 \%)$ \\
\hline LRTI with RV16, n (\% of confirmed) & $10(77 \%)$ & $8(73 \%)$ \\
\hline \multicolumn{3}{|c|}{$\begin{array}{l}{ }^{*} \mathrm{p}<0.05 \text { ( } \chi^{2} \text { test). } \\
{ }^{* * *} \mathrm{p}<0.001 \text { (Mann-Whitney } \mathrm{U} \text { test). } \\
+\mathrm{C} \text { onfirmation by PCR or RV16 neutralisation. } \\
\text { BAL, bronchoalveolar lavage; FEV } \% \text { pred, forced expiratory volume in } 1 \mathrm{~s} \text { expressed as } \\
\text { percentage of the predicted value; } \mathrm{LRTI} \text {, lower respiratory tract infection based on } \\
\text { positive PCR in bronchial brush, BAL cells or BAL fluid; } \mathrm{PC}_{20} \text {, provocative methacholine } \\
\text { concentration causing a } 20 \% \text { fall in FEV } \mathrm{F}_{1} ; \mathrm{RV} 16 \text {, rhinovirus type } 16 \text {. }\end{array}$} \\
\hline
\end{tabular}

in BAL fluid $(\mathrm{r}=0.7824, \mathrm{p}<0.0001)$ were even stronger (figure 5). Similar correlations were observed when subjects without PCR-confirmed lower respiratory tract infections were excluded (see online supplementary figure S3).

\section{DISCUSSION}

In the present study we showed that a low-dose rhinovirus infection $\left(10 \mathrm{TCID}_{50}\right)$ induced characteristics of a mild exacerbation in patients with asthma, reflected by increased asthma symptoms, reduced lung function and enhanced eosinophilic inflammation. Although IDO activity did not change upon rhinovirus infection, patients with allergic asthma already showed lower IDO activity in the lungs at baseline than healthy individuals. Conversely, enhanced levels of cytotoxic metabolites and tryptophan were found in the serum of patients with asthma which were associated with eosinophilic airway inflammation and peak asthma symptom scores after rhinovirus infection. There was no association between tryptophan catabolism in the airways and exacerbation parameters. 

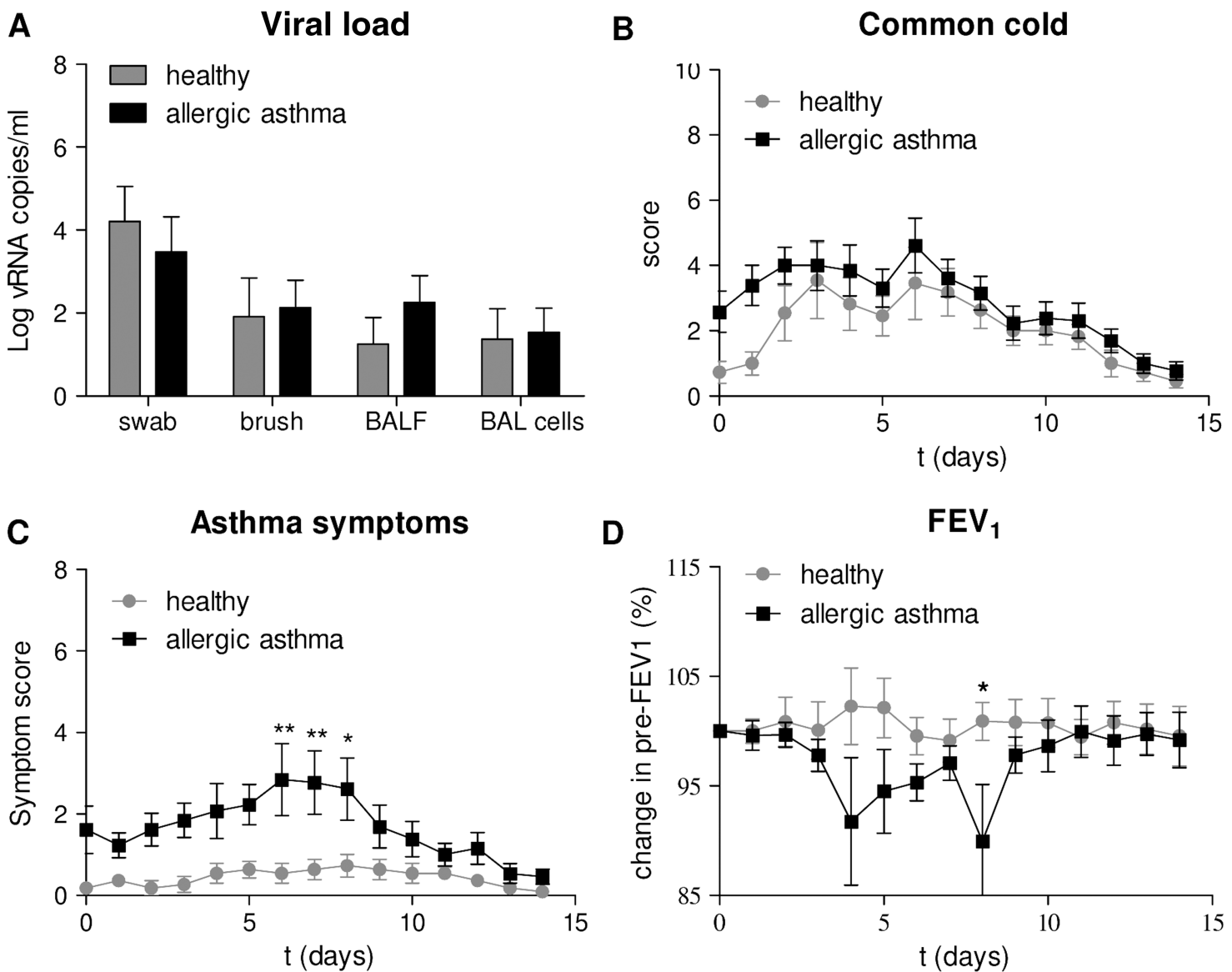

Figure 3 Virology and symptoms after experimental rhinovirus infection. (A) Viral RNA copies were determined in upper respiratory tract (nasal swabs) and lower respiratory tract (bronchial brushes, bronchoalveolar lavage (BAL) fluid (without cells) and pelleted BAL cells). (B) Common cold scores, $(C)$ asthma symptom scores and $(D)$ changes in forced expiratory volume in $1 \mathrm{~s}\left(\mathrm{FEV}_{1}\right)$ were reported daily. All data are mean $\pm \mathrm{SE}$. ${ }^{*} \mathrm{p}<0.05$ and ${ }^{* *} p<0.01$ versus healthy individuals.

Tryptophan is catabolised via multiple pathways. Tryptophan 2,3-dioxgenase (TDO), IDO1 and IDO2 are known to enhance the degradation of tryptophan into $\mathrm{N}$-formyl-kynurenine, which is rapidly converted into kynurenine ${ }^{27} 28$ and further degraded into 3-hydroxy-kynurenine, anthranilic acid, 3-hydroxy-anthranilic acid and quinolinic acid. TDO is constitutively and specifically expressed in the liver and maintains circulating tryptophan levels within a physiological range. ${ }^{29}$ In contrast, IDO1 and IDO2 are hardly expressed under normal conditions but are induced by inflammatory mediators. ${ }^{8-11} 29$ We and others have previously shown that viral airway infection leads to pulmonary expression of IDO. ${ }^{30-32}$

The lower levels of kynurenine and quinolinic acid in EBC indicate that pulmonary IDO activity is impaired in patients

Table 2 Inflammatory parameters in bronchoalveolar lavage fluid

\begin{tabular}{|c|c|c|c|c|}
\hline & \multicolumn{2}{|l|}{ Healthy individuals } & \multicolumn{2}{|c|}{ Allergic asthma patients } \\
\hline & Day -1 & Day 6 & Day -1 & Day 6 \\
\hline Eosinophils (\%) & $0.25(0.0-2.3)$ & $0.25(0.0-4.1)$ & $1.0(0.2-9.0)$ & $1.2(0.3-9.9)$ \\
\hline Neutrophils (\%) & $2.3(0.5-8.7)$ & $1.2^{*}(0.4-3.1)$ & $0.85(0.1-4.0)$ & $1.5(0.3-9.2)$ \\
\hline Macrophages (\%) & $87.2(69.9-97.0)$ & 88.9 (77.9-96.1) & $84.5(71.0-93.5)$ & $85.2(75.3-93.8)$ \\
\hline Lymphocytes (\%) & $6.9(2.4-28.0)$ & $8.3(3.1-21.2)$ & $10.6(4.8-23.1)$ & $10.3(4.9-17.0)$ \\
\hline $\mathrm{ECP}(\mathrm{pg} / \mathrm{mL})$ & 159 (77-698) & $174(111-459)$ & $286(136-3852)$ & $507^{*}(153-6336)$ \\
\hline MPO (ng/mL) & $4.16(0.5-16.45)$ & $3.85(0.5-11.93)$ & $4.04(0.5-11.83)$ & $4.16(0.5-17.87)$ \\
\hline IL-8 (pg/mL) & $0.9(0.07-10.55)$ & $1.25(0.10-4.04)$ & $0.34(0.00-2.33)$ & $1.42^{* *}(0.04-15.75)$ \\
\hline FeNO (ppb) & $17.5(7.6-44.0)$ & $16.5(9.0-58.0)$ & $58.7(24.5-138.6)$ & $72.2(28.0-164.1)$ \\
\hline
\end{tabular}


Serum
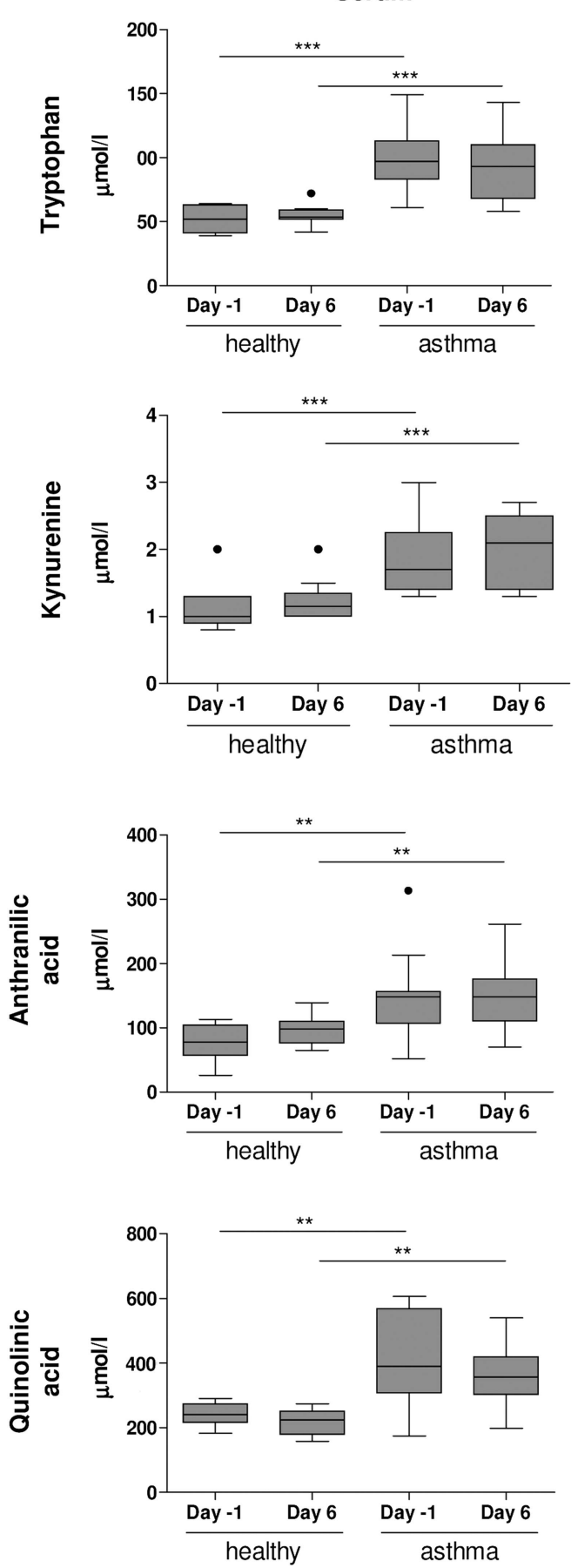

EBC
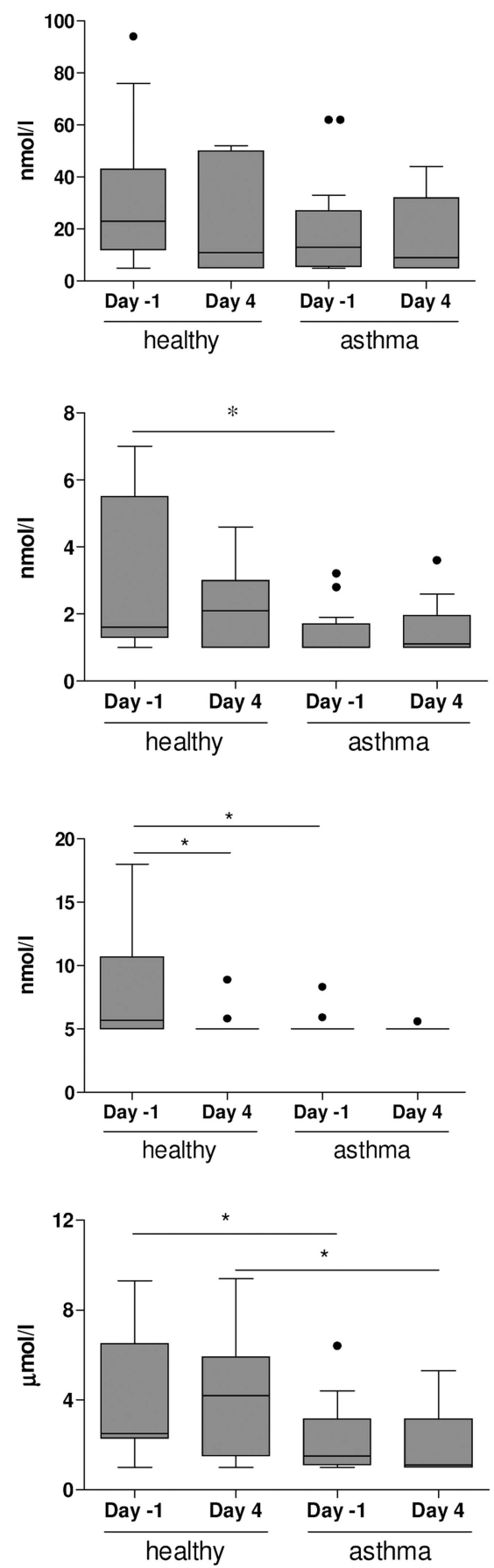

Figure 4 Tryptophan metabolome in exhaled breath condensate (EBC) and serum. Tryptophan, kynurenine, anthranilic acid and quinolinic acid were determined in serum (day -1 and day 6 ) and EBC (day -1 and day 4 ) of healthy individuals and patients with allergic asthma. Concentrations are shown as $\mathrm{nmol} / \mathrm{L}$ or $\mu \mathrm{mol} / \mathrm{L}$ (mean $\pm \mathrm{SE}$ ). ${ }^{*} p<0.05,{ }^{* *} p<0.01$ and ${ }^{* * *} p<0.001$ versus healthy individuals (Bonferroni-adjusted $p$ values). Kynurenine (day $-1, p=0.0186$ ), anthranilic acid (day $-1, p=0.0197$ ) and quinolonic acid (day $4, p=0.0166$ ) were lower in $E B C$ from patients with

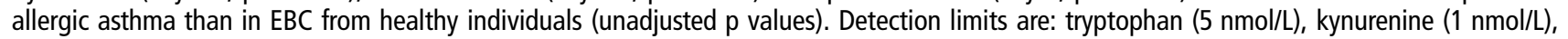
quinolinic acid (1 $\mathrm{nmol} / \mathrm{L})$ and anthranilic acid $(5 \mathrm{nmol} / \mathrm{L})$.

with allergic asthma. This reduced IDO activity in patients with allergic asthma may relate to reduced IDO expression but also to nitric oxide, which is known to bind the heme group of IDO $^{33}$ thereby limiting its enzymatic activity. Of note, patients with allergic asthma have higher fractions of exhaled nitric oxide.

In contrast to our expectation, rhinovirus infection did not enhance IDO activity in either healthy individuals or those with asthma. This observation may be explained in several mutually non-exclusive ways. First, the observation that kynurenine and quinolinic acid are present in EBC indicates that IDO is already expressed in the pulmonary compartment under normal conditions, which may have masked a modest effect induced by rhinovirus infection. Second, the volunteers were challenged with a low dose of RV16. Despite increased common cold and asthma symptoms, the inflammatory response may have been 

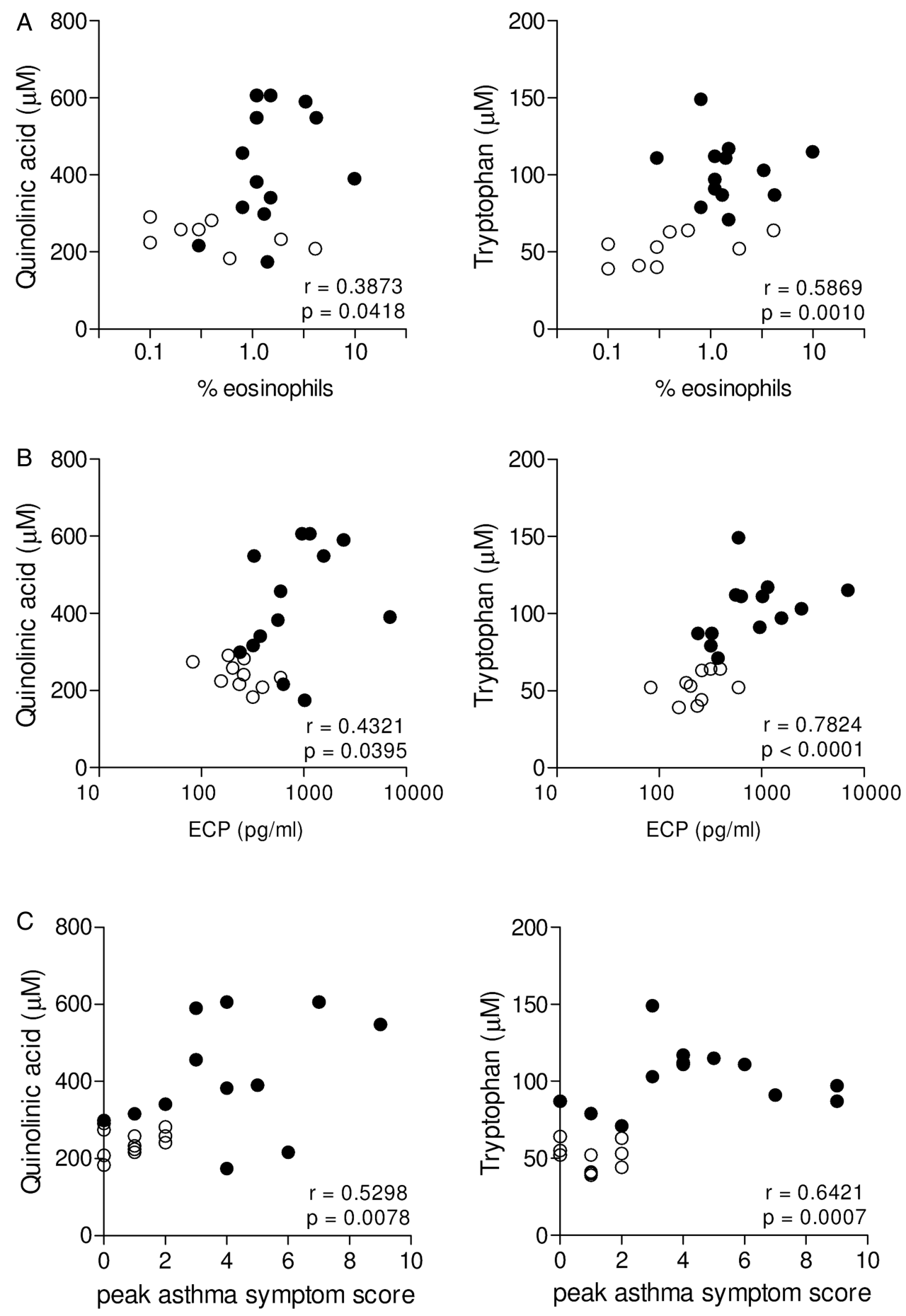

Figure 5 Association between tryptophan metabolism and eosinophilic inflammation. Analyses of the correlation between systemic quinolinic acid (left) and systemic tryptophan (right) with (A) percentage eosinophils in bronchoalveolar lavage (BAL) fluid, (B) eosinophil cationic protein concentration in BAL fluid and (C) peak asthma symptom scores after rhinovirus exposure in patients with asthma (solid dots) and healthy individuals (open dots). Spearman correlation coefficients $(r)$ and $p$ values are given in each panel.

insufficient to induce IDO expression. RV16 showed an increase in ECP and IL-8 in BAL fluid of individuals with asthma, but mediators that have been described to modulate IDO expression were not detectable. It should be noted that low-dose rhinovirus exposure resulted in similar common cold and asthma symptom scores as those described for high-dose rhinovirus exposure, ${ }^{5}$ 1826 which argues against the low-dose rhinovirus as a possible explanation. In addition, we only included subjects with confirmed viral infection-that is, subjects without neutralising antibodies against RV16 - at the start of the study. The fact that animal studies showing pulmonary IDO expression have been performed with influenza ${ }^{30-32}$ might indicate that the virulence of the virus is more relevant for pulmonary IDO expression. Prospective observational studies are required to determine whether influenza induces IDO in human airways.

In serum, patients with allergic asthma showed significantly higher levels of tryptophan and cytotoxic metabolites than healthy individuals, which confirms previous observations by 
Warraki et al. ${ }^{34}$ Interestingly, we observed a significant association between quinolinic acid and percentage eosinophils and ECP in BAL fluid. These findings indicate that quinolinic acid may be used as a marker for local eosinophilic inflammation in response to rhinovirus infection. The higher tryptophan levels in serum from patients with allergic asthma is in line with a higher OR of having asthma with high tryptophan levels in plasma as reported by Fogarty et al. ${ }^{35}$ It should be noted that this study only included patients who received daily inhaled steroids whereas our study only included patients without inhaled or oral corticosteroids. Whether the enhanced systemic levels of kynurenine catabolites in patients with asthma have physiological consequences such as reduced $\mathrm{T}$ cell responses remains to be determined.

For markers of arginine metabolism we obtained a similar systemic increase as previously shown by Lara et al. ${ }^{25}$ In line with our findings for quinolinic acid, we observed lower arginine levels in EBC from patients with asthma. Healthy individuals but not those with asthma showed increased ornithine levels in EBC upon rhinovirus challenge, which may point towards a role for arginase and/or alternatively activated macrophages in host defence towards viral airway infection in healthy individuals. Together these findings indicate that the amino acid catabolism in the systemic and pulmonary compartments can be quite distinct.

In the present study we exposed volunteers with a 1000-fold lower dose than in previous studies on rhinovirus-induced asthma exacerbations. ${ }^{6} 18 \quad 26$ Although common cold and asthma symptoms peaked 2 days later, the symptoms scores were similar to those described for the high viral inoculum. Twenty-four individuals $(86 \%)$ had a confirmed viral infection as reflected by detection of virus by quantitative PCR on day 6 or RV16 neutralisation between days 42 and 56 after viral infection. These data indicate that low-dose RV16, which is more likely to reflect a natural common cold than high-dose RV16, can be used to provoke common cold and asthma symptoms in patients with mild allergic asthma. The fact that we did not observe differences in viral load between healthy individuals and those with asthma must be interpreted with caution since viral load was determined at a single time point.

The design of this study has some important limitations. First, the male/female ratio was different for healthy individuals (1/13) and patients with asthma (7/7), which might have an impact on the results. However, there were no differences between men and women with asthma with respect to systemic amino acid levels. In addition, the differences between healthy individuals and those with asthma were still significant when only female volunteers were included in the statistical analysis. We therefore conclude that the male/female ratio is not a substantial confounding factor.

Second, the healthy individuals and those with asthma differed in bronchial hyperresponsiveness to methacholine and also in allergy status. We specifically chose to select for allergies in the asthma group since we hypothesised that the balance between IFN $\gamma$ and Th2 cytokines would affect the expression of IDO and, hence, also tryptophan catabolism. Since these cytokines were undetectable or hardly detectable in BAL fluid, we were unable to observe an association between cytokine responses and tryptophan turnover. It is therefore not possible to conclude whether the differences between healthy individuals and patients with allergic asthma are due to allergy, asthma or a combination of both. Further research is required to identify the contribution of the allergic status.
Third, volunteers underwent bronchoscopy 1 day before exposure to RV16. This procedure probably affected common cold scores and asthma symptom scores, particularly for individuals with allergic asthma. Nonetheless, both healthy individuals and patients with allergic asthma showed a significant increase in common cold symptoms and, in addition, patients with asthma showed a significant increase in asthma symptoms. These findings indicate that rhinovirus infection was successful in these volunteers. It is important to note that blood and EBC samples were always collected before the bronchoscopic procedure. The second EBC sample was collected on day 4 and the second blood sample on day 6. Differences between EBC and plasma are therefore only mentioned if these differences were already present on day 1 prior to infection when EBC and blood were collected in parallel.

In conclusion, the present study shows that patients with mild allergic asthma have lower IDO activity in the pulmonary compartment than controls, which is not enhanced by experimental rhinovirus infection. Systemic levels of tryptophan and downstream metabolites of TDO/IDO-mediated tryptophan degradation were markedly higher in patients with allergic asthma and were associated with eosinophilic inflammation and asthma symptom scores during experimental rhinovirus infection. The underlying cause of these newly observed differences in tryptophan metabolism remains to be elucidated.

\section{Author affiliations}

${ }^{1}$ Department of Respiratory Medicine, Academic Medical Center, University of Amsterdam, Amsterdam, The Netherlands

${ }^{2}$ Department of Experimental Immunology, Academic Medical Center, University of Amsterdam, Amsterdam, The Netherlands

${ }^{3}$ Department of Laboratory of Experimental Intensive Care and Anesthesiology, Academic Medical Center, University of Amsterdam, Amsterdam, The Netherlands

${ }^{4}$ Department of Genetic Metabolic Disorders, Academic Medical Center, University of Amsterdam, Amsterdam, The Netherlands

${ }^{5}$ Department of Medical Microbiology, Academic Medical Center, University of Amsterdam, Amsterdam, The Netherlands

${ }^{6}$ Airway Disease Infection Section, National Heart and Lung Institute, Imperial College London, London, UK

${ }^{7}$ Department of Respiratory Medicine, Onze Lieve Vrouwe Gasthuis, Amsterdam, The Netherlands

Acknowledgements We would like to thank Katrien Grünberg (VU Medical Center, Amsterdam, the Netherlands), José de Kluijver (Leiden University Medical Center, Leiden, the Netherlands) and Joseph Footitt (Imperial College, London, UK) for advice on the rhinovirus infection protocol and the members of the RESOLVE research team: Marieke Berger, Niki Fens, David Yick, Ariane H Wagener, Marijke Amelink, Herre J Reesink, Paul Bresser, Peter W A Kunst, Lisette N Venekamp, Christof J Majoor, Reindert P van Steenwijk, René E Jonkers. We would also like to thank Dr A H Zwinderman for advice on statistical analysis of the data.

Collaborators Marieke Berger; Niki Fens; David Yick; Ariane H Wagener; Marijke Amelink; Herre J Reesink; Paul Bresser; Peter W A Kunst; Lisette N Venekamp; Christof J Majoor; Reindert P van Steenwijk; René E Jonkers.

Contributors KFvdS was involved in the conception and design of the study, acquisition, analysis and interpretation of the data and wrote the manuscript. MAvdP, WK, AD, HWvE, JAK, RM and KCW were involved in acquisition, analysis and interpretation of the data. SLJ, JSvdZ, PJS and RL were involved in the conception and design of the study and interpretation of the data. All authors have seen and approved the content of the manuscript prior to submission. The RESOLVE research team members were actively involved in clinical aspects related to the conduct of this study.

Funding This study was financially supported by a grant from the Netherlands Asthma Foundation (grant no 3-2-07-012). SLJ is supported by ERC FP7 advanced grant 233015, a Chair from Asthma UK (CH11SJ) and MRC Centre grant G1000758.

\section{Competing interests None.}

Ethics approval The study was approved by the Medical Ethics Committee of the Academic Medical Center in Amsterdam, The Netherlands and registered at the Netherlands Trial Registration (no.1677). 
Patient consent Obtained.

Provenance and peer review Not commissioned; externally peer reviewed.

\section{REFERENCES}

1 Reddel HK, Taylor DR, Bateman ED, et al. An official American Thoracic Society/ European Respiratory Society statement: asthma control and exacerbations. Standardizing endpoints for clinical asthma trials and clinical practice. Am J Respir Crit Care Med 2009;180:59-99.

2 Papadopoulos NG, Xepapadaki P, Mallia P, et al. Mechanisms of virus-induced asthma exacerbations: state of the art. A Ga2len and InterAirways document. Allergy 2007;62:457-70.

3 Johnston SL, Pattemore PK, Sanderson G, et al. Community study of role of viral infections in exacerbations of asthma in 9-11-year-old children. BMJ 1995;310:1225-9.

4 Murray CS, Simpson A, Custovic A. Allergens, viruses, and asthma exacerbations. Proc Am Thorac Soc 2004;1:99-104.

5 Mallia P, Johnston SL. How viral infections cause exacerbation of airway diseases. Chest 2006;130:1203-10.

6 Message SD, Laza-Stanca V, Mallia P, et al. Rhinovirus-induced lower respiratory illness is increased in asthma and related to virus load and Th1/2 cytokine and IL-10 production. Proc Natl Acad Sci USA 2008;105:13562-7.

7 MacKenzie CR, Gonzalez RG, Kniep E, et al. Cytokine mediated regulation of interferon-gamma-induced IDO activation. Adv Exp Med Biol 1999;467:533-9.

8 Chaves AC, Ceravolo IP, Gomes JÁ, et al. IL-4 and IL-13 regulate the induction of indoleamine 2,3-dioxygenase activity and the control of Toxoplasma gondii replication in human fibroblasts activated with IFN-gamma. Eur J Immunol 2001;31:333-44

9 Adams $\mathrm{O}$, Besken $\mathrm{K}$, Oberdorfer $\mathrm{C}$, et al. Inhibition of human herpes simplex virus type 2 by interferon gamma and tumor necrosis factor alpha is mediated by indoleamine 2,3-dioxygenase. Microbes Infect 2004;6:806-12.

10 Terajima M, Leporati AM. Role of indoleamine 2,3-dioxygenase in anti-viral activity of interferon-gamma against vaccinia virus. Viral Immunol 2005;18:722-9.

11 Bodaghi B, Goureau O, Zipeto D, et al. Role of IFN-gamma-induced indoleamine 2,3 dioxygenase and inducible nitric oxide synthase in the replication of human cytomegalovirus in retinal pigment epithelial cells. J Immunol 1999:162:957-64.

12 Hwu P, Du MX, Lapointe R, et al. Indoleamine 2,3-dioxygenase production by human dendritic cells results in the inhibition of T cell proliferation. J Immunol 2000;164:3596-9.

13 Lee GK, Park HJ, Macleod M, et al. Tryptophan deprivation sensitizes activated T cells to apoptosis prior to cell division. Immunology 2002;107:452-60.

14 Hayashi T, Beck L, Rossetto $C$, et al. Inhibition of experimental asthma by indoleamine 2,3-dioxygenase. J Clin Invest 2004;114:270-9.

15 Taher YA, Piavaux BJ, Gras R, et al. Indoleamine 2,3-dioxygenase-dependent tryptophan metabolites contribute to tolerance induction during allergen immunotherapy in a mouse model. J Allergy Clin Immunol 2008;121:983-9.

16 von Bubnoff D, Fimmers R, Bogdanow M, et al. Asymptomatic atopy is associated with increased indoleamine 2,3-dioxygenase activity and interleukin-10 production during seasonal allergen exposure. Clin Exp Allergy 2004;34:1056-63.
17 Mallia P, Message SD, Kebadze T, et al. An experimental model of rhinovirus induced chronic obstructive pulmonary disease exacerbations: a pilot study. Respir Res 2006;7:116.

18 Grünberg K, Sharon RF, Sont JK, et al. Rhinovirus-induced airway inflammation in asthma: effect of treatment with inhaled corticosteroids before and during experimental infection. Am J Respir Crit Care Med 2001;164:1816-22.

19 Hoogerwerf JJ, de Vos AF, Levi M, et al. Activation of coagulation and inhibition of fibrinolysis in the human lung on bronchial instillation of lipoteichoic acid and lipopolysaccharide. Crit Care Med 2009;37:619-25.

20 Jansen RR, Wieringa J, Koekkoek SM, et al. Frequent detection of respiratory viruses without symptoms: toward defining clinically relevant cutoff values. J Clin Microbiol 2011;49:2631-6.

21 Reed LJ, Muench H. A simple method of estimating fifty percent endpoints. Am J Hyg 1938;27:s493-7.

22 Boorsma M, Lutter R, van de Pol MA, et al. Repeatability of inflammatory parameters in induced sputum of COPD patients. COPD 2007:4:321-9.

23 Rijssenbeek-Nouwens $\mathrm{LH}$, Fieten $\mathrm{KB}$, Bron AO, et al. High altitude treatment in atopic and non-atopic patients with severe asthma. Eur Respir $J$ 2012:40:1374-80.

24 Horváth I, Hunt J, Barnes PJ, et al. ATS/ERS Task Force on Exhaled Breath Condensate. Exhaled breath condensate: methodological recommendations and unresolved questions. Eur Respir J 2005;26:523-48.

25 Lara A, Khatri SB, Wang Z, et al. Alterations of the arginine metabolome in asthma. Am J Respir Crit Care Med 2008;178:673-81.

26 de Kluijver J, Grünberg K, Pons D, et al. Interleukin-1beta and interleukin-1ra levels in nasal lavages during experimental rhinovirus infection in asthmatic and non-asthmatic subjects. Clin Exp Allergy 2003;33:1415-18.

27 Naismith JH. Tryptophan oxygenation: mechanistic considerations. Biochem Soc Trans 2012;40:509-14.

28 Lob S, Konigsrainer A, Schafer R, et al. Levo- but not dextro-1-methyl tryptophan abrogates the IDO activity of human dendritic cells. Blood 2008;111:2152-4.

29 Wirleitner B, Neurauter G, Schröcksnadel K, et al. Interferon-gamma-induced conversion of tryptophan: immunologic and neuropsychiatric aspects. Curr Med Chem 2003:10:1581-91.

30 Jacoby DB, Choi AM. Influenza virus induces expression of antioxidant genes in human epithelial cells. Free Radic Biol Med 1994;16:821-4.

31 van der Sluijs KF, Nijhuis M, Levels JH, et al. Influenza-induced expression of indoleamine 2,3-dioxygenase enhances interleukin-10 production and bacterial outgrowth during secondary pneumococcal pneumonia. J Infect Dis 2006;193:214-22.

32 Yoshida $\mathrm{R}$, Urade $\mathrm{Y}$, Tokuda $\mathrm{M}$, et al. Induction of indoleamine 2,3-dioxygenase in mouse lung during virus infection. Proc Natl Acad Sci USA 1979;76:4084-6.

33 Hara $\mathrm{T}$, Ogasawara $\mathrm{N}$, Akimoto $\mathrm{H}$, et al. High-affinity uptake of kynurenine and nitric oxide-mediated inhibition of indoleamine 2,3-dioxygenase in bone marrow-derived myeloid dendritic cells. Immunol Lett 2008;116:95-102.

34 Warraki SE, el-Gammal MY, el-Asmar MF, et al. Serum kynurenine in bronchial asthma and chronic bronchitis. Chest 1970;57:148-50.

35 Fogarty A, Broadfield E, Lewis $\mathrm{S}$, et al. Amino acids and asthma: a case-control study. Eur Respir J 2004;23:565-8. 